Presence of Cancer-producing Substances in Roasted Food

A SERIES of mice was painted every second day for a long time in the ordinary manner in the occipital region with an alcohol or petroleum extract $(a)$ of horse muscle, treated at a temperature of $275^{\circ} \mathrm{C}$., (b) of browned butter, or (c) of roasted coffee. As controls we employed $(a)$ a series of mice painted in the same manner with an alcohol or petroleum ether extract of horse muscle dried on a water bath or in a hot current of air, untreated butter and unroasted coffee, and $(b)$ a number of untreated animals.

The experimental series have so far comprised 241 animals-1 149 males and 92 females. The spontaneous frequency of malignant tumours among the females is 10 per cent at most. Among the males this frequency is, as usual, much lower.

So far, no malignant tumours have been observed in the male mice painted with extract of either roasted or unroasted food. On the other hand, a giant-cell spindle-cell sarcoma was produced in the painted region in a series of mice which had been painted for a long time with pure cholesterol, treated at $275^{\circ} \mathrm{C}$., emulsified in coco-nut fat. Further, certain chronic changes of great interest, which will be described later, were observed in the animals treated with roasted food.

Twenty-three of the female mice treated with extracts of roasted food survived a period of application of 11 months. In this period we observed nine malignant tumours in the mammary glands, all of the adenocarcinoma type ( $=40$ per cent $)$, namely, two after the application of petroleum ether extract of roasted coffee, one after browned butter, two after petroleum ether extract of roasted muscle and four after the application of an alcohol extract of roasted muscle. The average time for the appearance of these tumours was 360 days. In the control series (26 animals) only one case of mammary cancer (= 4 per cent) was observed during an observation period of 378 days.

The statistical probability of such a distribution of the tumours (9 tumours in 23 animals treated with an extract of roasted material and 1 in 26 animals in the control series) occurring simply by chance is not more than 0.0026 , that is, the chance of this occurring in such series is only one in 380 . Moreover, as the tumour frequency has increased to 40 per cent, as compared with the maximum expected incidence of 10 per cent, the probability that chance has played a part is much less, and on that account I consider that $I$ am justified in concluding from the results of these experiments that extracts of roasted food contain one or more carcinogenic substances.

These substances may be thought to reach the mammary tissue by resorption from the skin. However, very little change was noticed in the skin during the entire period of application. Cramer and Horning ${ }^{1}$ and Burrows ${ }^{2}$ have produced mammary cancer in mice by the application of estrone. Attempts to demonstrate the presence of an cestrogen factor in the extract, however, have not yet given any positive results. Another possibility is that the carcinogenic factor reaches the organism per os, as the animals very quickly lick off the painted extract. The isolation of the active substance is in progress. A full report will be published in the Proceedings of the Royal Physiographical Society of Lund.

The present investigation was suggested by the observations of Kennaway,4 and Kennaway and
Sampson ${ }^{5}$ that heating human skin, muscle, yeast and cholesterol in a hydrogen atmosphere to $700^{\circ}-$ $920^{\circ} \mathrm{C}$. produces carcinogenic substances with a local effect.

\section{E. M. P. Widmark.}

Institute of Medical Chemistry,

University of Lund, Sweden. April 15.

${ }^{1}$ Cramer, W., and Horning, E. S., Lancet, 230, 247 (1936).

'Burrows, H., Amer. J. Cancer, 24, 613 (1935).

${ }^{3}$ Kennaway, E. L., J. Path. Bact., 27, 233 (1924).

- Kennaway, E. L., Brit. Med. J., 11, 1 (1925).

${ }^{8}$ Kennaway, E. I., and Sampson, J. Path. Bact., 81, 609 (1928).

\section{Influence of Lactate on the Absorption of Glucose} from the Intestine of Adrenalectomized Rats

IN experiments designed to throw light on the mechanism of sugar absorption and its control by the adrenal cortex, it was previously found ${ }^{1}$ that with adrenalectomized rats sodium pyruvate restores the normal sugar absorption from the intestine. Similar experiments using the same methods hove now been carried out with sodium lactate which was administered continuously in doses of 15-20 mgm. a day subcutaneously. It was found that it shows the same effect as pyruvate does, restoring the low glucose absorption of the adrenalectomized rats to the normal level. (Normal absorption in forty-five minutes, 80.0 per cent of the injected glucose, with adrenalectomized rats $42 \cdot 3$ per cent, after lactate administration $73 \cdot 7$ per cent.)

\section{LaASZT.}

Physiological Laboratory,

University, Fribourg, Switzerland. May 1.

I Laszt, L., Verh. schweiz. Physiol., Feb. 1939.

\section{Relation between the Secretory Cells of the Pars Nervosa of the Hypophysis and Classical Neuroglia}

GERSH $^{1}$ has shown that the glial cells of the pars nervosa - the pituicytes of Bucy² - are responsible for the secretion of the anti-diuretic principle of the posterior lobe. I have presented additional evidence ${ }^{3}$ which demonstrates that these cells elaborate the pars nervosa hormones. By the use of silver carbonate methods for neuroglia, developed by the Spanish school, Bucy showed that the pituicytes, like neuroglial astrocytes in the central nervous system, possess cytoplasmic processes, one or more of which end on connective tissue either in the form of pia, of that around blood vessels, or of septa. The pituicytes, however, are impregnated most satisfactorily by methods which demonstrate consistently neuroglial oligodendrocytes (these have cytoplasmic processes but no vascular attachments in contradistinction to astrocytes) and mesodermal microglia. Astrocytes are usually not impregnated by these methods.

In order to establish a clearer concept of the relationship between pituicytes and neuroglia, I have compared the morphology, genesis, and cytoplasmic inclusions of pituicytes with those of neuroglia. 